Cartagena, 7 a 9 de septiembre de 2011

\title{
La Matriz de Operaciones y Materiales y la Matriz de Operaciones y Recursos, un nuevo enfoque para resolver el problema GMOP basado en el concepto del Stroke
}

\section{The Operations \& Materials Matrix and the Operations \& Resources Matrix, a new approach to solve the GMOP problem based on the Stroke concept}

\author{
Julien Maheut y José P. Garcia-Sabater \\ ROGLE - Departamento de Organización de Empreses. Universitat Politécnica de Valencia. Camino de Vera s/n, 4602 I Valencia. \\ juma2@upvnet.upv.es.jpgarcia@omp.upv.es
}

Fecha de recepción: 7-9-2011

Fecha de aceptación: 19-9-2011

\begin{abstract}
Resumen: La gestión de materiales de productos multi-nivel usa desde los años 1970 la metodología denominada MRP. La incorporación del CRP en los ERP conjuntamente al MRP tradicional dio nacimiento a los llamados MRPII. Sin embargo, desde aquella época en la literatura, se asigna una única lista de materiales y una lista única de recursos a cada producto susceptible de ser ensamblado lo que implica fuertes limitaciones para considerar operaciones alternativas. En el problema GMOP, se propone el uso del concepto del stroke para resolver el problema de planificación de las operaciones y de los materiales considerando operaciones alternativas. En este artículo, se presenta primero una evolución del problema GMOP introduciendo las recepciones programadas, y segundo, se presenta el despliegue y análisis de las matrices que se proponen para facilitar la implementación de un modelo y una herramienta que se basen en el concepto de stroke.
\end{abstract}

Palabras clave: cadena de suministro, planificación de las operaciones, operaciones alternativas, stroke, MRP, GMOP.

\begin{abstract}
Since the 1970, the multi-stage product materials management used the methodology called MRP. The CRP incorporation in the traditional ERP jointly with the MRP gave birth to the so-called MRPII. However, since that time in the literature, a unique bill of materials and a unique list of resources are assigned to each product to be assembled which implies strong limitations to consider alternative operations. In the GMOP problem, the use of the stroke concept is proposed to solve the operations and materials planning problem considering alternative operations. The aim of this paper is to present firstly an evolution of the GMOP problem considering scheduled receptions, and secondly to present the deployment and analysis of the matrices that are proposed to facilitate a model and tool implementation based on the stroke concept.
\end{abstract}

Keywords: supply chain, operations planning, alternative operations, stroke, MRP, GMOP.

\section{Introducción}

La gestión de materiales de productos multi-nivel usa desde los años 1970 la metodología denominada Material Requirement Planning (MRP). Esta metodología impulsada por Orlicky (1975) que se basa únicamente en la planificación de los materiales con lista de materiales directas tuvo que evolucionar para hacer frente a las necesidades cada vez más complejas de las distintas industrias. Una de las evoluciones más relevantes fue la incorporación del Capacitated Res- source Planning en los Enterprise Ressource Planning conjuntamente al MRP tradicional para incorporar las limitaciones de capacidad y el routing (Plenert, 1999) en los Ilamados MRPII (Wight, 1984). Billington et al. (1983) propusieron plantear el MRP capacitado mediante programación matemática. Evidentemente las limitaciones tecnológicas les impedían afirmar que el modelo sin más fuera aplicable y en el mismo artículo proponen métodos para resolver el problema. El planteamiento de los autores consistía en asignar una única lista de materiales y una lista úni- 
ca de recursos a cada producto susceptible de ser ensamblado. Esta estructura se ha mantenido en la literatura desde entonces sin modificaciones.

La matriz que vincula cada producto padre (parent item) con los componentes que son necesarios para ensamblarlo (child items) aparece en esa formulación. Esta matriz se denominó Gozinto (Vazsonyi, 1954). En (Billington et al., 1983), se incorpora el tiempo de entrega (Lead-Time) que se asocia también al producto, así como un yield a la producción, y la lista de recursos también en forma de matriz. Pero no se incorpora diferentes rutas para producir un mismo producto o tampoco se considera la posibilidad de usar listas inversas o alternativas de producción así como la posibilidad de trabajar entre dos niveles de una cadena teniendo en cuenta alternativas de transporte.

En este artículo, se pretende proponer el uso de una nueva estructura que reemplaza la tradicional lista de materiales y la lista de recursos. El modo de construcción y la interpretación de dicha estructura, basada en tres o cuatro matrices, permite la planificación de las operaciones en estricto nivel de igualdad a la de requerimientos de materiales. Es de destacar que dichas matrices permiten planificar las operaciones teniendo en cuenta todas las estructuras posibles de productos, las rutas alternativas, las alternativas en cuanto a las Operaciones (que sean de aprovisionamiento, de transformación, de venta o de transporte) pero también permiten una fácil integración para el caso de redes de suministro multi-sitio y permiten considerar operaciones de apoyo como es el caso del mantenimiento.

Este artículo presenta una doble contribución. En primer lugar se presenta una evolución del problema Generic Materials \& Operations Planning (Garcia-Sabater et al., 20।2) en el que se introduce las recepciones programadas debido a los strokes que ya se están ejecutando a principio del horizonte de planificación. Se aborda explícitamente el problema de planificación de una red de suministro multi-sitio y se incluye la consideración de las recepciones planificadas. Además se propone una nomenclatura para los productos que incluya de manera consistente no sólo el nombre o número de referencia de cada artículo sino también su embalaje, su ubicación y la cantidad de unidades que van en ellos. La segunda aportación es el despliegue y análisis de las matrices que se proponen para facilitar la implementación de un modelo y una herramienta que se basen en el concepto de stroke.
El resto del artículo se ha estructurado como sigue. En el segundo apartado, se describe brevemente el concepto del stroke que se plantea para la planificación de las operaciones y se aporta una caracterización del stroke que extiende la presentada en (Garcia-Sabater et al., 2012). En el tercer apartado, se presenta el modelo propuesto del llamado problema GMOP (Generic Materials \& Operations Planning) que se basa en el concepto del stroke incorporando los efectos del lead-time. Para resolver el problema GMOP, se necesita como mínimo el uso de dos matrices que se introducen y se caracterizan: la matriz de Operaciones \& Recursos que se presenta en el cuarto apartado y la matriz de Operaciones \& Materiales que se presenta en el quinto apartado. En el sexto apartado, se analiza la estructura para un problema multi-sitio y un caso práctico de aplicación. Finalmente, se presentan las conclusiones y algunas líneas futuras de investigación identificadas.

\section{El concepto de Stroke}

Los productos que se consideran en el problema GMOP son, o pueden ser, SKUs (Stock Keeping Units en inglés), es decir productos en su embalaje y su ubicación, y también se consideran los recursos que se tienen que planificar. Se asume que cada stroke puede necesitar un producto o un conjunto de productos localizados (o ningún producto en casos determinados) en un posible embalaje determinado que se consumen durante dicho stroke. A estas entradas, se las denomina stroke inputs y la cantidad de cada SKU consumido se denomina stroke input factor (concepto similar al factor de Gozinto). Al conjunto de productos (si existe) que se obtiene mediante la ejecución de un stroke determinado, se le denomina stroke output. Y la cantidad de cada SKU que se genera depende del stroke output factor.

Una definición de un stroke sería la siguiente:

«Un stroke representa cualquier operación básica (en su sentido más genérico), tarea o actividad. Puede transformar, transportar o consumir un conjunto de productos (medido preferentemente como SKU) para obtener o generar otro conjunto de productos (también medido preferentemente en SKU). Cada stroke puede utilizar o inmovilizar recursos en su ejecución.»

Los recursos que se consideran pueden ser de diferente naturaleza (maquinaria, recursos humanos, medios de transporte) pero no se pueden mover entre ubicaciones. En el problema GMOP aquí presentado 
los recursos no se adquieren o se venden durante el horizonte de planificación: están o no disponibles. Estos recursos, a los cuales se pueden asociar una capacidad, se vinculan directamente a cada stroke y no al producto (o conjunto de productos) que se obtiene como se puede apreciar en la Figura I. Se pueden obtener parcialmente de la Lista de Recursos como en (Tatsiopoulos, 1996).

Dicha operación, y por tanto el stroke que la representa, puede tener costes asociados (como por ejemplo coste de setup y/o coste unitario de ejecución), plazo de entrega asociado, y puede consumir una cierta cantidad de recursos a partir del primero de los periodos de planificación del plazo de entrega.

Para poder estructurar los datos de forma sencilla, se propone a continuación el uso de una nomenclatura para definir los SKUs:

- El producto «O I » almacenado en la planta A será denominada P0I@A mientras que el mismo producto ubicado en la planta B se denominará POI@B.

- El rack «0 I» con 12 productos «02» en él y ubicado en la planta B se denominará R0 I \# I 2P 02@A.

- El rack «0 I » vacío en la planta $C$ se denominará Rol\#00@C.

En el ejemplo presentado en la Figura I, el stroke $S_{k 1}$ tiene 3 inputs y 2 outputs. Los stroke inputs son:Y unidades de P0I@A, Z unidades de P02@A y I unidad de R0I\#XP03@A. Los outputs que se generan cuando se ejecuta una unidad de $S_{k l}$ son $X$ unidades de P03@A y I unidad de R0I\#YP0IZP02@A. Para la realización de una unidad de este stroke, un leadtime, unos costes así como la utilización de recursos se consideran.

Las hipótesis que se asumirán para el problema GMOP son las siguientes:

- El consumo en recursos de un stroke se representa en un periodo completo de planificación.

- El consumo en recursos de un stroke se limitará al primer periodo de la ejecución del mismo stroke.

- Un recurso no puede cambiar de localización. Esto puede implicar, si es necesario, que un recurso de transporte (por ejemplo un camión) se tenga que considerar como un SKU.

- La cantidad de strokes que se planifican en un periodo de tiempo es un entero positivo.

- Un stroke tiene que tener siempre algún dato asociado no nulo para ser válido.

\section{El problema GMOP}

El modelo GMOP que se presenta a continuación no se limita a presentar el modelo presentado en (Garcia-Sabater et al., 2012) sino que se incorpora el efecto del lead-time sobre las ecuaciones de continuidad

Figura I

Representación conceptual de un Stroke Sk

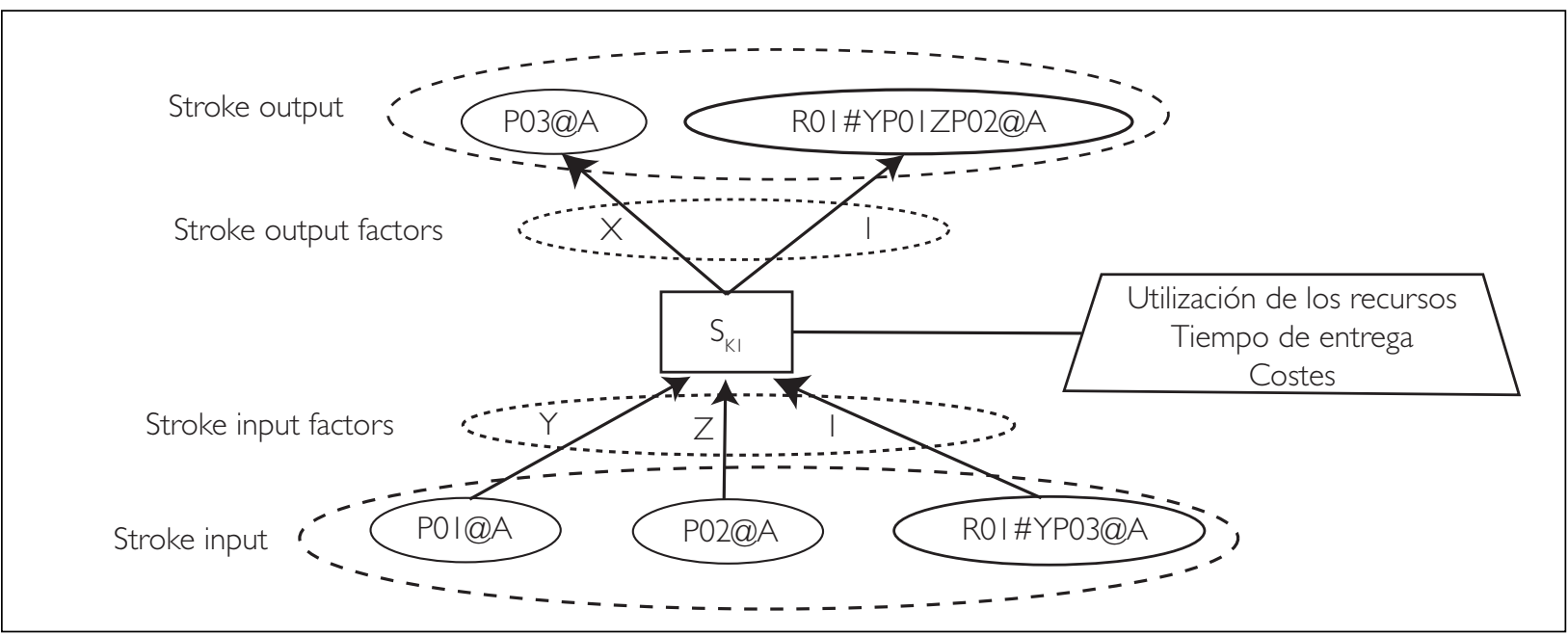

Dirección y Organización, Núm. 45, diciembre 20II | ISSN (On line): 2 I7I-6323 - ISSN (Print): II 32- I75X 
del inventario y además considera los niveles iniciales de inventario. Resolviendo así una de las principales limitaciones en su implementación práctica que aquel modelo tenía. La notación del modelo de programación matemática se presenta en la Tabla I. El problema GMOP se formula como un modelo de programación entera mixta.

El objetivo [ I] busca la minimización de los costes de setup de los strokes, de los costes unitarios de stroke y de los costes de almacenamiento. La ecuación [2] representa la continuidad de los niveles de inventario de los SKUs i. El nivel de inventario al final de un periodo considera el nivel de inventario al final del periodo anterior, las recepciones planificadas (debido a strokes en proceso), la demanda del producto y el consumo y la producción de SKUs debido a la ejecución de strokes. Debido a la existencia de lead-times no nulos, se considera en [3] la ecuación de continuidad para los primeros periodos. Con la

Tabla 1

Notación para el problema GMOP

\begin{tabular}{|c|c|}
\hline \multicolumn{2}{|c|}{ Índices y conjuntos } \\
\hline $\begin{array}{l}i \in P=\{1, \ldots, p\} \\
r \in R=\{1, \ldots, m\} \\
k \in S=\{1, \ldots, n\} \\
t=1, \ldots, T \\
L_{x} \subseteq S \\
S_{r} \subseteq S\end{array}$ & $\begin{array}{l}\text { SKUs } \\
\text { Recursos } \\
\text { Strokes } \\
\text { Periodos } \\
\text { Conjunto de strokes cuyo lead-time es inferior a } X \in\{0, \ldots, l\} \\
\text { Conjunto de strokes que utilizan el recurso } r\end{array}$ \\
\hline \multicolumn{2}{|l|}{ Parámetros } \\
\hline \multicolumn{2}{|r|}{$\begin{array}{l}\text { Demanda en } S K U \text { i durante en el periodo } t \\
\text { Coste de almacenar una unidad de } S K U \text { i durante el periodo } t \\
\text { Tiempo de operación para la ejecución de una unidad de stroke } k \text { en el recurso } r \\
\text { Tiempo de setup del stroke } k \text { en el recurso } r \\
\text { Coste de planificar la ejecución de una unidad de stroke } k \text { durante el periodo } t \\
\text { Coste de setup del stroke } k \text { durante el periodo } t \\
\text { Nivel inicial de inventario del } S K U i \\
\text { Recepciones planificadas en } S K U \text { s } i \text { durante el periodo } t \\
\text { Capacidad disponible del recurso } r \text { durante el periodo } t \\
\text { Número de unidades de } S K U s \text { s resultado de la ejecución de una unidad de stroke } k \text { (stroke output factor) } \\
\text { Número de unidades de } S K U s \text { s que se consumen durante la ejecución de una unidad de stroke } k \text { (stroke input factor) } \\
\text { Lead time de un stroke } k\end{array}$} \\
\hline \multicolumn{2}{|l|}{ Variables } \\
\hline \multicolumn{2}{|c|}{$\begin{array}{l}\text { Cantidad de strokes } k \text { que empiezan durante el periodo } t \\
\text { Nivel de inventario del SKU } i \text { al final del periodo } t \\
\text { Vale I si el stroke } k \text { está en set up durante el periodo } t \text { ( } 0 \text { en caso contrario) }\end{array}$} \\
\hline
\end{tabular}

Minimizar $F(z, l, \delta)=\sum_{t=1}^{T}\left(\sum_{i \in P}\left(h_{i t} \cdot l_{i t}\right)+\sum_{k \in S}\left(p_{k t} \cdot z_{k t}+\delta_{k t} \cdot f_{k t}\right)\right)$

Sujeto a

$$
\begin{array}{ll}
I_{i t}=I_{i, t-1}+Y_{i t}-d_{i t}+\sum_{k \in S}\left(S O_{i k} \cdot z_{k, t-L T}\right)-\sum_{k \in S}\left(S I_{i k} \cdot z_{k, t}\right) & i \in P, t=1+L, \ldots, T \\
I_{i, t}=I_{i, t-1}+Y_{i, t}-d_{i t}+\sum_{k \in L_{t-1}}\left(S O_{i k} \cdot z_{k, t-L T}\right)-\sum_{k \in S}\left(S I_{i k} \cdot z_{k, t}\right) & i \in P, t=L, \ldots, I \\
z_{k t}-M \cdot \delta_{k t} \leq 0 & k \in S, t=1, \ldots, T \\
\sum_{k \in S_{r}}\left(\delta_{k t} \cdot S t_{k r}+z_{k t} \cdot o t_{k r}\right) \leq K_{r t} & r \in R, t=1, \ldots, T \\
I_{i t} \geq 0 & i \in P, t=1, \ldots, T \\
z_{k, t} \geq 0, \delta_{k t} \in\{0,1\} & k \in S, t=1, \ldots, T
\end{array}
$$


restricción [4], si se produce un stroke en el periodo $t$, se asigna un valor no nulo a la variable que representa la existencia de setup. La restricción [5] representa la limitación de la capacidad productiva en cada periodo para cada recurso. Las ecuaciones [6][7] definen el dominio de definición de las variables.

Como se puede apreciar en el modelo GMOP, planificar usando la variable stroke resulta muy diferente a la planificación tradicional que se basa en la matriz Gozinto y la lista de recursos ya que lo que se planifica es el stroke (la operación, la tarea o la actividad). Expresado en otros términos, el stroke es la variable de decisión mientras que los materiales se generan y/o consumen en función de la ejecución de los strokes, por tanto no se planifica la producción del material sino las operaciones que se llevan a cabo para obtenerlos.

Debido a la necesidad de trabajar con una nueva representación de los datos, se propone en el apartado siguiente una descripción de la matriz de Operaciones \& Recursos y en otro apartado, se propone la descripción de la Matriz de Operaciones \& Materiales.

\section{La Matriz de Operaciones \& Recursos}

\section{I. Construcción de la matriz de Operaciones \& Recursos}

La matriz de Operaciones \& Recursos es la matriz que asigna a cada stroke los diferentes recursos que se inmovilizan o utilizan y el valor de la utilización del conjunto de recursos asociados. Esta matriz se construye en función una matriz de asignación de recursos a strokes que se denotará como Rs. Esta matriz $R^{S}=\left[r_{k r}\right]$ es una matriz de asignación binaria (en el sentido que $r_{k r} \in\{0,1\}$ para todos los $k$ y $r$ ) como se puede observar en la Tabla 2.

Tabla I

\section{Ejemplo de una matriz RS}

\begin{tabular}{|c|c|c|c|}
\hline$r_{k r}$ & 1 & 2 & 3 \\
\hline 1 & 1 & 0 & 0 \\
\hline 2 & 0 & 1 & 0 \\
\hline 3 & 1 & 0 & 1 \\
\hline 4 & 0 & 0 & 0 \\
\hline
\end{tabular}

Gracias a la matriz $R^{S}$, se puede construir la matriz que asigna a cada par stroke-recurso el valor del consumo de tiempo asociado.

\subsection{Ejemplo de una matriz de Operaciones \& Recursos}

Como en la mayoría de los problemas reales, un stroke usa un número muy limitado de recursos, la matriz de Operaciones \& Recursos consta de mucho elementos nulos. Para facilitar la construcción de la matriz, se propone en la tabla 3 el ejemplo de una matriz «sparse» que define el consumo de recursos de diferentes strokes.

Tabla 3

Ejemplo de una matriz sparse de Operaciones \& Recursos

\begin{tabular}{|c|c|c|c|}
\hline Stroke $k$ & Recurso $r$ & $\begin{array}{c}\text { ot } t_{k r} \text { (unidades } \\
\text { de tiempo) }\end{array}$ & $\begin{array}{c}s t_{k r} \text { (unidades } \\
\text { de tiempo) }\end{array}$ \\
\hline 1 & 1 & 14 & 12 \\
\hline 2 & 2 & 13 & 1,14 \\
\hline 3 & 1 & 0,7 & 200 \\
\hline 3 & 3 & 100 & 44 \\
\hline
\end{tabular}

Usar la matriz de Operaciones \& Recursos permite considerar rutas alternativas pero también permite considerar strokes que no consumen ningún tipo de recurso. Si consideramos la Tabla 3, asumiremos que el recurso $r \mid$ es el recurso «máquina $\mid », r 2$ el recurso «máquina 2» y el recurso r3 la «máquina 3». Se puede entonces interpretar esta matriz:

- El stroke $k l$ consume capacidad de un único recurso $\mathrm{rl}$ y el stroke $\mathrm{k} 2$ consume capacidad de un único recurso $r 2$. En este caso, los strokes $k l$ y k2 son strokes básicos en cuanto a recursos.

- Se puede considerar el caso en el cual se agregan las operaciones tal como es el caso del stroke k3. Este stroke no solamente consume tiempo del recurso $\mathrm{rl}$ sino también del recurso $\mathrm{r} 3$. Si el recurso $r 3$ fuera de mano de obra, el stroke consideraría al mismo tiempo un consumo de tiempo de máquina y un tiempo de mano de 
obra, lo que puede resultar interesante en casos determinados.

- Pueden existir casos en los cuales un stroke no consume ningún recurso planificado (caso del stroke k4). No significa por tanto que el stroke no consume ningún recurso, sino que no es necesaria la planificación de los recursos consumidos por cualquier motivo. En la matriz de Operaciones \& Recursos, tendríamos en ese caso $\sum_{r} r_{r, k}=0$.

Es relevante observar que los costes asociados a los strokes no pertenecen a esta matriz. Los costes asignados a los strokes dependen únicamente del stroke y del periodo considerado y contienen directamente el coste por consumir tiempo de los diferentes recursos. Y el tiempo de entrega afecta únicamente al stroke pero no a su recurso asociado. Del mismo modo que el lead-time sólo afectará al stroke.

\section{La Matriz de Operaciones \& Materiales}

\subsection{Construcción de la matriz de Operaciones \& Materiales}

La matriz de Operaciones \& Materiales representa para cada stroke el valor de los stroke outputs y de los stroke inputs asociado con el uso de una matriz única. Esta matriz que escribiremos $S^{S}=\left[s_{k i}\right]$ se forma de elementos naturales enteros. Se compone de elementos positivos asociados a los outputs y valores negativos asociados a los inputs.

Esta representación no se puede usar para el uso directo en modelos de programación matemática cuando existe un lead time no nulo para un stroke. Sin embargo, se puede transformar esta matriz de forma sencilla para su uso efectivo en el modelo GMOP. Esta matriz se divide en dos matrices positivas tal que $S^{S}=\left[S_{k i}\right]=S O-S I=\left[S_{k i}\right]-\left[S_{k i}\right]$ asumiendo que $s_{k i}$ y $s i_{k i}$ sean elementos naturales positivos para cualquier valor del índice $i$ o $k$.

El valor de $\mathrm{so}_{\mathrm{ki}}$ es el stroke output factor presentado en el segundo apartado. El valor de $s_{k i}$ es el stroke input factor.

Con el fin de poder describir la matriz $S^{S}$, se presentan nuevas notaciones en la Tabla 4.

El índice $j$ hace referencia a una localización definida geográficamente o a un miembro en particular de una red de suministro. El índice $p$ hace referencia a producto (con su embalaje) pero sin tener en cuenta su localización. En teoría, un producto $p$ disponible en una localización jl y el mismo disponible en una localización j2 son dos productos distintos. Por esta razón, usaremos el índice $p(j)$ para distinguir $p(j 1)$ de $p(j 2)$. Observar que con el índice i que se plantea en la Tabla l, ya se contempla esta distinción entre estos dos productos pero el análisis de las matrices resulta difícil de explicar. En función de esta caracterización se puede determinar la naturaleza de los diferentes strokes que se pueden considerar y realizar una comparación de los strokes entre ellos.

Tabla 4

Nuevos índices para caracterizar la Matriz

\begin{tabular}{|ll|}
\hline \multicolumn{1}{|l|}{ 'ndices } & \\
\hline$j \in J=\{j l, \ldots, j n\}$ & $\begin{array}{l}\text { Ubicaciones/Localizaciones/Plantas con- } \\
\text { sideradas }\end{array}$ \\
$p \in L=\{p l, \ldots, p n\}$ & Producto en su embalaje sin considerar \\
& la ubicación \\
$p(j)$ & Producto $p$ en la localización $j$ \\
$k(j)$ & Stroke $k$ que se ejecuta en la localización $j$ \\
\hline
\end{tabular}

\section{de Operaciones \& Materiales}

\subsection{La naturaleza de los strokes}

$$
\text { Stroke de Compra }
$$

Un stroke de compra es un stroke que no considera ningún stroke input pero que tiene por lo menos un stroke output asociado a la ejecución de este. De esta forma, se puede caracterizar un stroke de compra $k$ l de la forma siguiente:

$$
\begin{aligned}
& \sum_{i} s O_{i, k l}>0 \\
& \sum_{i} s i_{i, k l}=0
\end{aligned}
$$

Stroke de transformación

Un stroke de transformación puede ser una operación de ensamblaje, de desmontaje, de (des)empaquetado, o una transformación química. Estas operaciones deben ser localizadas en el sentido que el producto no puede cambiar de ubicación. Así, se puede caracterizar un stroke de transformación $k I(j \mid)$ de la forma siguiente:

$$
\sum_{p(j \mid)} s O_{p(j \mid), k \mid(j \mid)}>0
$$




$$
\begin{gathered}
\sum_{p(1))} s i_{p(1), k(j)}>0 \\
\sum_{p(1)} s O_{p(2), k(j))}=\sum_{p(2)} s i_{p(2), k(j)}=0, \quad \forall j 2 \neq j 1
\end{gathered}
$$

Los strokes que incluyen la gestión de los embalajes, se asimilarán a strokes de transformación. Los embalajes se pueden dividir en dos categorías, los embalajes retornables (como las paletas) y los embalajes duraderos que se usan a los largo de los procesos de fabricación/transporte. En cualquier caso, esos productos se consumen (por uso) y se producen (con procesos de desembalaje) a los largos de los procesos productivos sin ninguna regla en particular.

\section{Stroke de destrucción y stroke de venta}

Planificar las operaciones usando el concepto del stroke permite considerar también de forma uniforme strokes de destrucción de productos. La destrucción de productos es un hecho básico en la logística inversa y puede ocasionar costes asociados. Este stroke considera productos como inputs pero ningún producto en output. En la matriz de Operaciones \& Materiales, el stroke $k$ de destrucción se representaría de la forma siguiente:

$$
\begin{aligned}
& \sum_{i} s o_{i, k}=0 \\
& \sum_{i} s i_{i, k}>0
\end{aligned}
$$

Los strokes de venta se pueden considerar de forma análoga a los strokes de destrucción ya que tienen inputs pero no tienen outputs.

\section{Stroke de transporte entre dos plantas}

Un stroke de transporte entre dos plantas es un stroke que transporta un $S K U p(j 1)$ en un $S K U p(j 2)$ con $j 2$ diferente de $j l$. En este caso, este stroke se representaría de la forma siguiente:

$$
\begin{aligned}
& {\left[s i_{p(1), k}\right]=\left[s O_{p(2), k}\right], \quad j 1 \neq j 2} \\
& \sum_{p(2)} s O_{p(2), k)}>0, \quad j \mid \neq j 2 \\
& {\left[s O_{p(1), k}\right]=\left[s i_{p(2), k}\right]=[0], \quad j \mid \neq j 2}
\end{aligned}
$$

Stroke de transporte entre de una planta hacia varias plantas

El caso de envíos agrupados se puede considerar también. Si los envíos se hacen desde $j$ l hasta $u=\{j 2, \ldots, j n\}$ pues se tendría el stroke $k(j l)$ siguiente:

$$
\begin{aligned}
& {\left[s O_{p(1), k(1)}\right]=\left[s i_{p(u), k(1)}\right]=[0]} \\
& s i_{p(I), k(1)}=\sum_{p(u)} s O_{p(u), k(1)}
\end{aligned}
$$

\section{Stroke de apoyo/decisión}

Otro ejemplo de stroke que se puede considerar es la inmovilización de un recurso o el consumo planificado de recursos durante un periodo dado. Un ejemplo práctico es la necesidad de planificar ventanas temporales de mantenimiento para ciertos recursos. En la matriz de Operaciones \& Materiales, este stroke no consideraría ni inputs ni outputs de forma que $s_{i, k}=0$ y $s_{i, k}=0$ para todo $i$ y $k$. Ese el caso por ejemplo de tareas de mantenimiento que se deben planificar en secuenciación o en planificación y que dejan inmovilizado a un recurso. También puede ser el caso de tiempos parados sin fabricación cuando por ejemplo se envían en formación a equipos de trabajo.

\subsection{Propiedades interesantes}

Gracias al uso de las matrices presentadas anteriormente, la mayor aportación destacable del uso de estas es que se puede considerar operaciones alternativas. A continuación, se presenta algunas de las posibles alternativas que se puede considerar.

\section{Strokes con inputs alternativos}

En la práctica, a veces las listas de materiales son «flexibles» en el sentido que una combinación alternativas de productos en inputs pueden ser utilizado para producir un conjunto de productos. En la literatura, se denominan alternative $\mathrm{BOMs}$, flexible BOMs, alternate $B O M s$, problemas de recetas, product sustitution o incluso transhipments (Maheut et al., 2012). Usando el concepto del stroke, los alternative BOMs son strokes con inputs alternativos. Un stroke $k 2$ con input substituto al stroke $k$ I tiene las características siguientes:

$$
\begin{aligned}
& {\left[\mathrm{so}_{i, k 1}\right]=\left[\mathrm{so}_{i, k 2}\right] \neq[0], k \mid \neq k 2} \\
& {\left[s i_{i, k 1}\right]=\left[s i_{i, k 2}\right], \quad k \mid \neq k 2}
\end{aligned}
$$

\section{Strokes con outputs alternativos}

Un stroke con output alternativo puede representar muchas operaciones o procesos en la realidad industrial. Por ejemplo en la química, se puede poner los mismos productos en condiciones distintas para obtener productos finales distintos. Otro ejemplo in- 
teresante es la inyección de piezas de plástico, los problemas de corte e inclusión las operaciones en prensas usando matrices distintas. En todos estos casos, los inputs de la operación pueden ser iguales y de la misma cantidad, pero al usar diferentes recursos o condiciones externas controladas, el resultado de la operación (es decir los outputs) será distinto. Las características matriciales serían las siguientes:

$$
\begin{aligned}
& {\left[\mathrm{so}_{i, k 1}\right]=\left[\mathrm{so}_{i, k 2}\right], \quad k 1 \neq k 2} \\
& {\left[s i_{i, k 1}\right]=\left[s i_{i, k 2}\right] \neq[0], \quad k \mid \neq k 2}
\end{aligned}
$$

Strokes alternativos con outputs e inputs no-nulos

Un stroke alternativo $k 1$ a otro stroke $k 2$ son dos strokes que tienen los mismos outputs y los mismos inputs. En este caso, se asume que por lo menos un input se consume y un output se genera al ejecutar los strokes para no considerar los strokes de compra o de venta.

Estos stroke se deben distinguir por sus matrices de Operaciones \& Recursos (si no, es un dato redundante). La característica que representan los strokes alternativos aparece en la literatura con los nombres de resource substitution y alternative routing. En la matriz de Operaciones \& Materiales, los vectores tienen las características siguientes:

$$
\left[s i_{i, k 1}\right]=\left[s i_{i, k 2}\right] \neq[0], \text { con } k \mid \neq k 2
$$

\section{Strokes alternativos de compra}

El uso del stroke permite también considerar strokes alternativos de compra. Un stroke $k l$ es stroke alternativo de compra a $k 2$ si los dos strokes tienen como outputs el mismo producto $p /$ pero que sus costes son diferentes, que sus stroke output factors (lote de compra) son diferentes, que los lead-times son diferentes o que los proveedores son diferentes.

De forma similar, existen strokes alternativos de ventas. Un stroke $k 3$ es stroke alternativo de venta a $k 4$ si los dos strokes tienen como inputs el mismo producto $p 2$ pero que sus costes son diferentes, que sus stroke input factors (lote de venta) son diferentes, que los lead-times son diferentes o que los proveedores son diferentes.

\section{Análisis de una Matriz de Operaciones \& Materiales genérica multi-sitio}

\section{I. Análisis de la estructura de la matriz de Operaciones \& Materiales genérica}

\section{en un caso de red multi-sitio}

Con el fin de poder entender la estructura que tiene todas las matrices de Operaciones \& Materiales, se propone en la Tabla 5 la introducción de nuevos conjuntos para facilitar el entendimiento de estas.

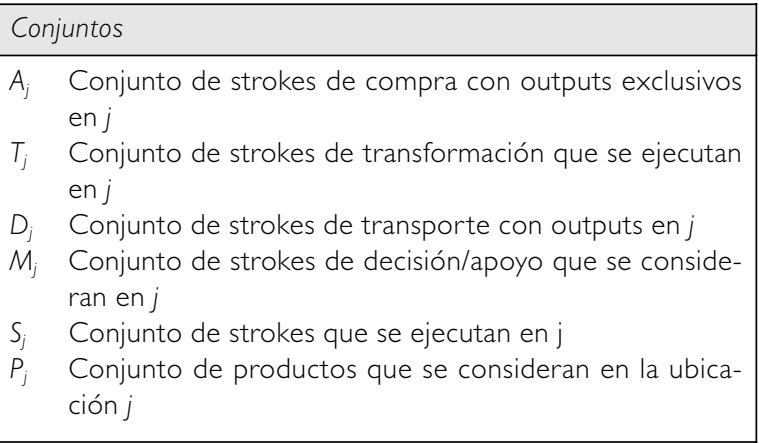

\section{Tabla 5}

\section{Conjuntos que permiten caracterizar la Matriz de Operaciones \& Materiales}

La matriz de Operaciones \& Materiales tiene una estructura básica en cuanto a las Operaciones que se realizan en una matriz multi-sitio. Esa estructura se basa en las operaciones básicas que se realizan y se planifican en la red de suministro. La estructura que se propone en la Figura 3 es un ejemplo de matriz para una red de suministro con 3 ubicaciones con envíos directos.

En la Figura 3, se observan dos aspectos interesantes:

- Se ve que los diferentes strokes son responsabilidad de una localización.

- La matriz de transporte D(jl) consiste en el conjunto de strokes cuyos outputs se ubican en $j l$. En el caso de la matriz de transporte asociado a j2 se consideran los strokes que no tienen outputs en $j$ l (y en el caso más general, en los niveles superiores de la cadena de suministro).

- La matriz entera consta con muchos bloques de datos nulos y bloques de datos que dependen de las diferentes áreas de planificación.

\subsection{Aplicación a un caso sencillo}

Para entender con datos reales el modo de construcción de una Matriz de Operación y Materiales, se propone un ejemplo sencillo que se presenta a continuación. 
Figura 3

Estructura de la matriz de Operaciones \& Materiales en función de las operaciones consideradas

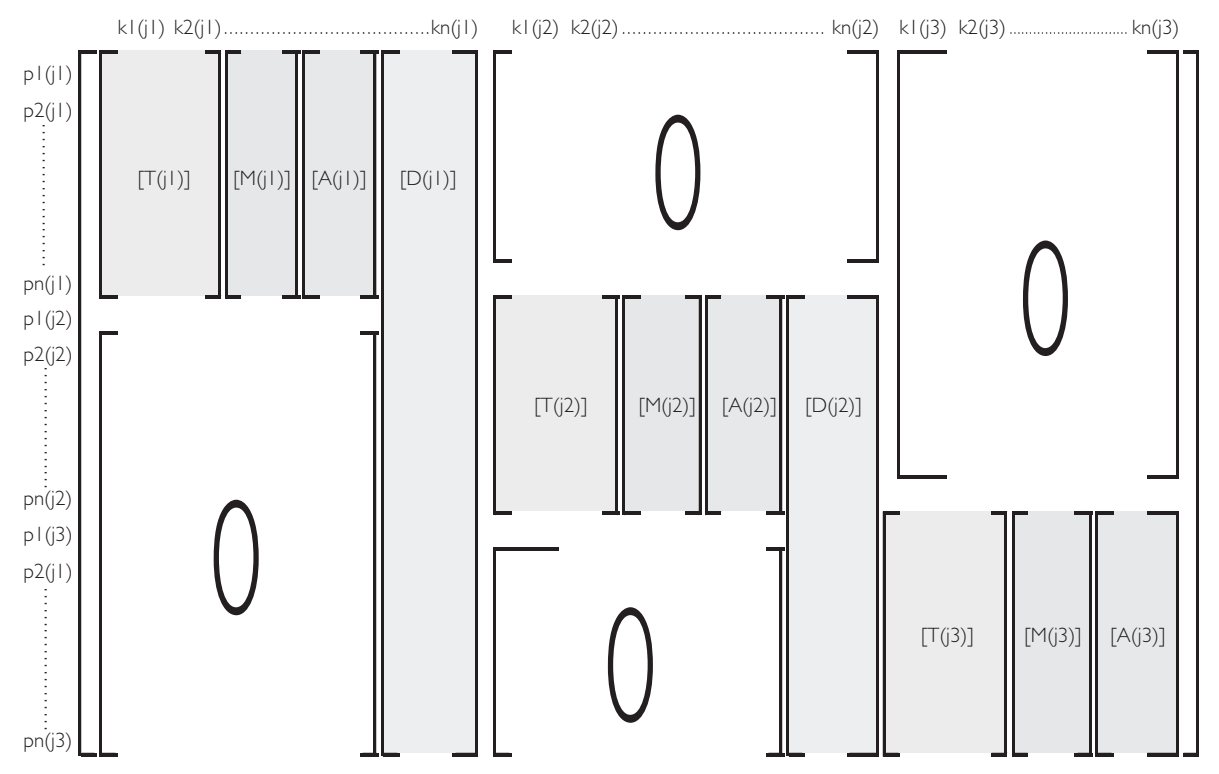

En este ejemplo, se consideran tres plantas en ubicaciones distintas ( $1, j 2$ y j3). La planta jl trabaja en Just In Sequence para un OEM del sector del automóvil. La planta j I recibe de la planta 2 racks llenos p6(jl) de productos. Un rack $p 6$ se compone de I rack vacio $p 8$ y de 8 productos $p 5$. La operación básica que consiste en desempaquetar los embalajes llenos es $k 5(j l)$. Una vez desempaquetado, los productos p5 se pueden colocan en un soporte de mecanización que debe tener 3 productos en él. Debido a un proceso productivo complejo, se puede colocar los productos $\mathrm{p} 5$ en dos soportes a la derecha (stroke $k 4(j l)$ ) o se pueden colocar 3 productos en un soporte a la izquierda y 3 otros en un soporte a la derecha con el stroke $k 3(j l)$. Si se realiza la operación $k 4$, se obtienen 2 productos $p 2(j l)$ mientras que se realiza $k 3(j l)$ se obtiene I $p 2(j l)$ y I p3(jl). El proceso de mecanización y la operación que consiste en quitar los productos acabo se representa con los strokes $k l(j l)$ y $k 2(j l)$. Estos dos últimos strokes se diferencian por la razón siguiente: en el primero, se considera la mecanización de un producto $p 3$ y un producto $p 4$ mientras que en el segundo, se mecaniza 2 productos p3. Esta línea de mecanización necesita un mantenimiento periódico con lo cual se debe tener en cuenta y se debe determinar cuándo se debe ejecutar este mantenimiento. Esta operación de mantenimiento se considera con el stroke $k 6(j l)$.
La planta j2 es una planta dónde se ejecutan operaciones de inyección y operaciones de ensamblaje. Las operaciones de inyección se realizan en dos prensas. Para realizar los productos $p 9(j 2)$ y $p / 0(j 2)$, la empresa puede usar una maquina I usando una matriz o usar la maquina 2 usando otra matriz diferente. Usando la maquinal, se consume 0.8 unidades de p 12(j2) para fabricar I unidad de p9(jl) y dos unidades de $p / 0(j)$. Por otro lado, si se usa la maquina2, consumiendo 1.2 unidad de $p / 2(j \mid 2)$, se obtiene I unidad de $p 9(j 1)$ y 4 unidades de $p / 0(j 2)$.

La segunda parte del proceso es el proceso de ensamblaje de productos. Para ensamblar una unidad de $p 5(j 2)$, se necesita una unidad de p9(j2), una de p I I (j2) y 3 unidades de $p / 0(j 2)$.

El producto p I I (j2) se puede comprar a un proveedor externo con un tamaño de lote de 50 o se puede transporte desde la planta j3. Esta última planta es capaz de producir el producto p / / (j3) con un tamaño de lote de 20 unidades.

Otra operación se deben considerar: los strokes de recirculación de racks vacíos, el transporte de racks llenos entre j2 y jl, el empaquetado de productos en j2 y los strokes de compra de p / 2(j2) y p / 2(j3).

La matriz de Operaciones y Materiales asociada al caso de estudiado presentado encima se presenta en la Figura 4 a continuación. 
Figura 4

Caso sencillo de una matriz de Operaciones \& Materiales

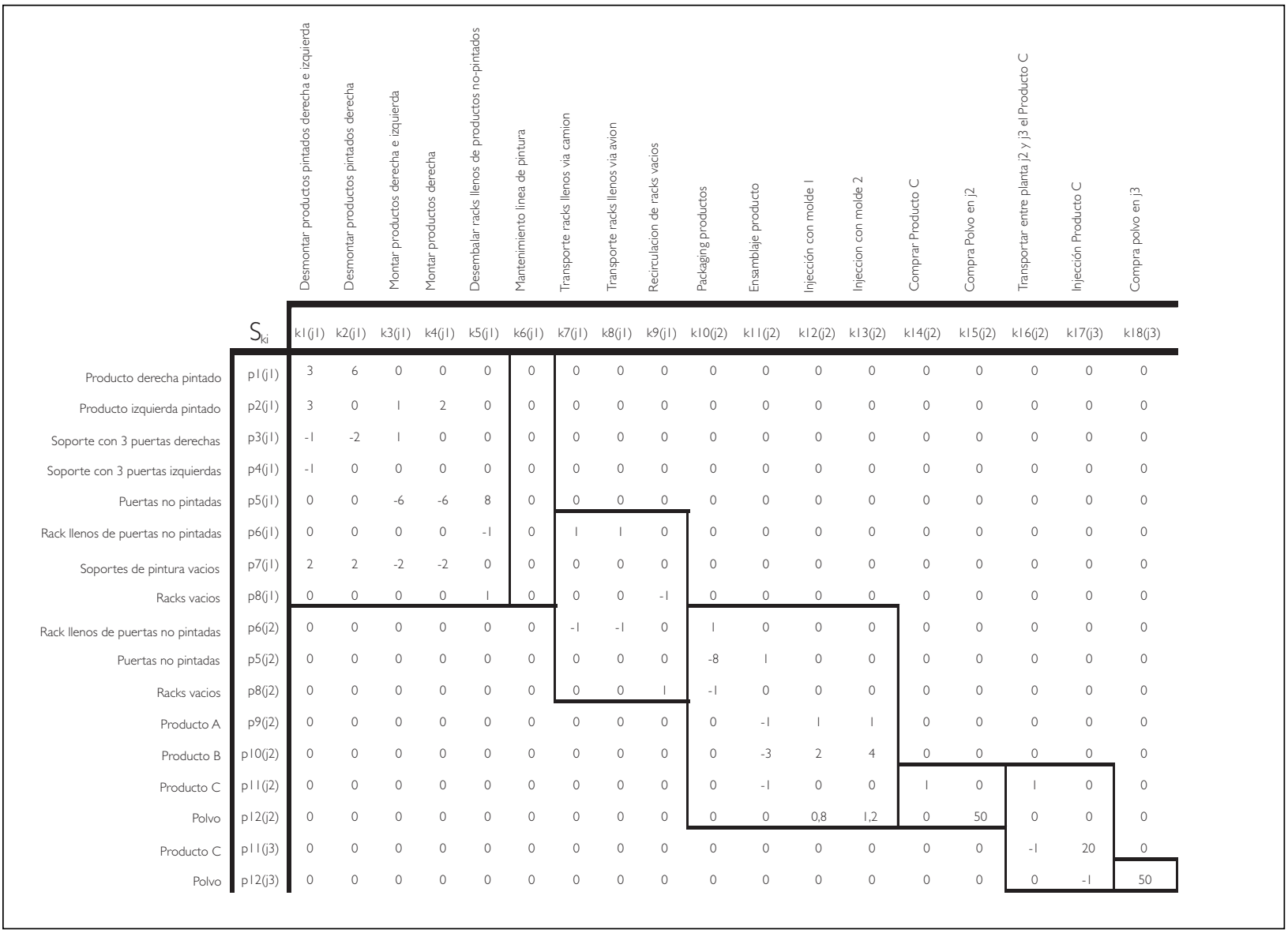

\section{Ventaja y desventajas \\ de la planificación con el uso de la variable stroke}

\section{I. Límites de la representación}

La construcción de estas matrices es sencilla pero a medida que vaya aumentado el número de productos, de operaciones, de recursos y de localizaciones el tamaño de las matrices irá creciendo. Y esto probablemente tendrá por consecuencia tiempos de carga de datos y tiempos de resoluciones más importantes.

\subsection{Uniformidad de la variable de decisión}

Como se puede apreciar en las figuras presentadas anteriormente, el uso del stroke permite representar de forma uniforme una gran variedad de problemas que se presentan en la planificación de operaciones. Basándose en una estructura más uniforme y sencilla con el concepto de stroke, el modelado matemático y la resolución del problema de planificación considera una única variable de decisión. Gracias a esta estructura es posible desarrollar algoritmos que se basen en un único vector homogéneo.

Como se observa en la Figura 5, las variables típicas de producción, compras, transporte o de operaciones diversas se pueden representar mediante una variable única que se puede representar en una tabla única con la variable $z_{k, t}$.

Esta uniformización de la variable de decisión permite limitar el número de tipos de variables en la función objetivo. Así también, aunque un stroke se caracterice por unos stroke outputs y strokes inputs, el modelo no necesita presentar mucho índices.

\section{Discusión, Conclusión y Líneas futuras de investigación}

En este trabajo, se ha presentado un modelo de programación matemática para la resolución del pro- 
Figura 5

Uniformización de la variable de decisión gracias al Stroke

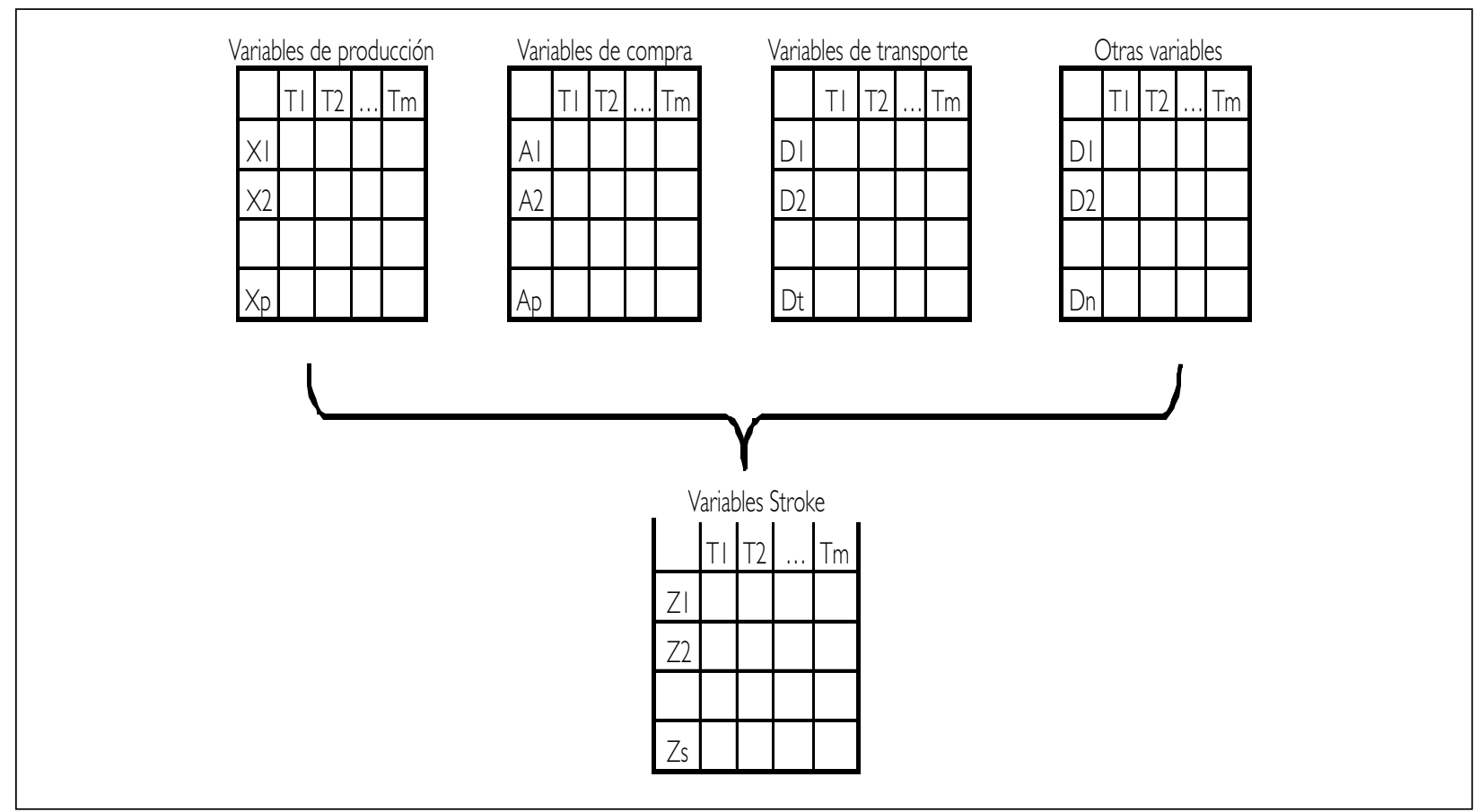

blema GMOP. Este problema se basa en el concepto «stroke» para la planificación de las Operaciones y de los Materiales en un nivel estricto de igualdad. En el modelo, se ha incorporado el efecto de los leadtimes sobre las ecuaciones de continuidad de los niveles de inventario.

Este nuevo enfoque implica la necesidad de trabajar con nuevas matrices diferentes a la matriz de Gozinto. En este trabajo, se propone la introducción de la matriz de Operaciones \& Materiales y el uso de la matriz de Operaciones \& Recursos que se basa en una matriz de asignación de strokes a recursos. Este trabajo propone un sencillo análisis de la matriz, presenta un caso sencillo de aplicación de las matrices. También se plantean las limitaciones y ventajas de este nuevo enfoque. Es de destacar que una de las principales ventajas del concepto de stroke y de las matrices introducidas es que permite un análisis estructurado del problema de planificación de las operaciones. También, se puede considerar alternativas de producción con una cierta facilidad ya que se puede introducir de forma estructurada nuevos datos.

Futuras líneas de investigación consisten en proponer procedimientos para la transformación de bases de datos tradicionales en bases de datos que soportan la planificación desde un punto de vista de las operaciones con el concepto del stroke. Otra línea de investigación consistirá en hacer experimentos para determinar el efecto de trabajar con el concepto de stroke sobre los tiempos de computación. También, resultará de gran interés analizar las estructuras de datos que hacen que el problema GMOP resulta más o menos difícil en su resolución. Y otra línea de investigación consistirá en apoyarse sobre estas novedosas estructuras de datos para resolver el problema distribuido de redes de suministro multi-sitio.

\section{Agradecimientos}

El presente trabajo se ha desarrollado gracias a la ayuda DPI2010-18243 del Ministerio de Ciencia e Innovación del Gobierno de España dentro del programa de Proyectos de Investigación Fundamental no orientada, con el título «Coordinación de operaciones en redes de suministro/demanda ajustadas, resilientes a la incertidumbre: modelos y algoritmos para la gestión de la incertidumbre y la complejidad». También, esta investigación ha sido parcialmente financiada por el Séptimo Programa Marco de la Comisión de las Comunidades Europea (FP7/20072013) mediante el acuerdo de subvención n. ${ }^{\circ}$ NMP2-SL-2009-229333, Proyecto «Resilient Multi-Plant Networks» (REMPLANET). Asimismo, esta investigación también ha sido financiada mediante 
una beca doctoral VALi+d concedida por la Generalitat Valenciana de España a Julien Maheut (Ref. ACIF/20I0).

\section{Bibliografía}

BILLINGTON, P.J., MCCLAIN, J.D., y THOMAS, L.J. ( 1 983), Mathematical programming approaches to capacityconstrained MRP systems: Review, formulation and problem reduction». Management Science, 29 (10), pp. ||26-||4|.

GARCIA-SABATER, J. P., MAHEUT, J., \& MARIN-GARCIA, J. A. A new formulation technique to model Materials and Operations Planning: the Generic Materials and Operations Planning (GMOP) Problem. European J.Industrial Engineering, (in press).

GARCIA-SABATER J.P., MAHEUT J., MARIN-GARCIA J.A., 2012 In press: «A Two-Stage Sequential Planning Scheme for Integrated Operations Planning and Scheduling System using MILP:The Case of an Engine Assembler»». Flexible Services and Manufacturing Journal, DOl: | 0.1007/s | 0696-0| |-9|26-z.
MAHEUT J., GARCIA-SABATER J.P., MULA F. 2012 In press, «A Supply Chain Operations Lot-Sizing and Scheduling Model with Alternative Operations», Industrial Engineering : Innovative Networks,S.P.Sethietal.(eds.), DOI: I 0. | 007/978- |-447| -232 | -7_35, Springer-Verlag London.

ORLICKY, J. (1975), Material Requirements Planning: The new way of life in production and inventory management. New York: McGaw Hill.

PLENERT, G. (1999), Focusing material requirements planning (MRP) towards performance». European Journal of Operational Research, I 9 ( I), pp. 91-99.

TATSIOPOULOS, I.P. (1996), On the unification of bills of materials and routings». Computers in Industry, 3 I (3), pp. 293-304.

VAZSONYI, A. ( 1954), The use of mathematics in production and inventory control». Management Science, I ( I), pp. 70-85.

WIGHT, O.W. (1984), Production and inventory management in the computer age. John Wiley \& Sons, Inc. New York, NY, USA. 